\title{
XVIII. Experiments for investigating the cause of the coloured concentric rings, discovered by Sir Isaac Newton, between two object-glasses laid upon one another
}

\author{
William Herschel LLD. F.R.S.
}

To cite this article: William Herschel LLD. F.R.S. (1808) XVIII. Experiments for investigating the cause of the coloured concentric rings, discovered by Sir Isaac Newton, between two object-glasses laid upon one another, Philosophical Magazine Series 1, 30:117, 72-90, DOI: 10.1080/14786440808563774

To link to this article: http://dx.doi.org/10.1080/14786440808563774

$$
\text { 曲 Published online: } 18 \text { May } 2009 .
$$

Submit your article to this journal ¿

\section{山ll Article views: 2}

Q View related articles ¿ 
probably a pair of bellows, constructed on purpase, will be contrived, so as to prevent unnecessary loss of the sulphur.

By the same friendly communication I have received fresh assurances, not only of the powerful influence of sulphur as a vermifuge against the whole tribe of worms and other insects, which infest, and prey upon, vegetables; but I also find, that in other respects this substance is even congenial to the health of those trees and plants on which it is sprinkled-that peach-trees in particular were remarkably improved by the sulphur; they absorbed it, and, it may be said, were even fond of it; for it was evideutly absorbed, and must have entered into the vegetable system. It was likewise noticed, that the verdure and other healthful appearances were perceptibly increased; for the quantity of new shoots and leaves formed subsequently to the operation, and having no sulphur on their surfaces, served as a kind of comparative index, and pointed out distinctly the accumulation of health.

Upon the whole, it may be observed, that, independently of its deleterious effects on the vermin, the question respecting these sanative powers of such an insoluble substance as sulphur, seems to be one of the utmost importance; and, I should think, must be highly interesting to the physiologist, and, indeed, to all men of science.

\section{I remain, sir,}

your obliged and obedient servant,

Long-Acre,

Feb. 24, 1808.

Jos. Hume.

XVIII. Experiments for investigating the Cause of the coloured concentric Rings, discovered by Sir IsaAC NewTon, letween two Object-glasses laid upon one another. By William Heaschel, LLD. F.R.S.*

The account given by Sir $I$. Newton, of the coloured arcs and rings which he discovered by laying two prisms or ob.

* From Philosophical Transactions for 1807, Part II. 
ject-glasses upon each other, is highly interesting. He very justly remarks, that these phænomena are "s of difficult consideration," but that "they may conduce to further discoveries for completing the theory of light, especially as to the constitution of the parts of natural bodies on which their colours or transparency depend *."

With regard to the explanation of the appearance of these coloured rings, which is given by Sir I. Newton, I must confess that it has never been satisfactory to me. He accounts for the production of the rings, by ascribing to the rays of light certain fits of easy reflection and easy transmission alternately returning and taking place with each ray at certain stated intervals $\uparrow$. But this, without mentioning particular objections, seems to be an hypothesis which cannot be easily reconciled "with the minuteness and extreme velocity of the particles of which these rays, according to the Newtonian theory, are composed.

The great beauty of the coloured rings, and the pleasing appearances arising from the different degrees of pressure of the two surfaces of the glasses against each other when they are formed, and especially the importance of the subject, have often excited my desire of inquiring further into the cause of such interesting phænomena; and with a view to examine them properly 1 obtained, in the year 1792, the two object-glasses of Huygens, in the possession of the Royal Society, one of 122 the other of 170 feet focal length, and began a series of experiments with them, which, though many times interrupted by astronomical pursuits, has often been taken up again, and has lately been carried to a very considerable extent. The conclusions that may be drawn from them, though they may not perfectly account for all the phænomena of the rings, are yet sufficiently well supported, and of such a nature as to point out several modifications of light that have been totally overlooked, and other: that have never been properly discriminated. It will, therefore, be the aim of this paper to arrange and distinguish the various modifications of light in a clear and perspicuous

* Newton's Optics, 4th ed. p. 169. + Ibid. p. 256.

order, 
order, and afterwards to give my sentiments upon the cause of the formation of the concentric rings. The avowed intricacy of the subject *, however, requires, in the first place, a minute detail of experiments, and afterwards a very gradual development of the consequences to be deduced from them.

As the word modification will frequently be used, it may not be amiss to say, that when applied to light, it is intended to stand for a general expression of all the changes that are made in its colours, direction, or motion : thus, by the modification of reflection, light is thrown back ; by that of refraction, it is bent from its former course; by the modification of dispersion, it is divided into colours, and so of the rest.

\section{Of different Methods to make one Sel of concentric Rings visible.}

In the beginning of my experiments I followed the Newtonian example, and having laid the two object-glasses of Huygens upon one another I soon perceived the concentric rings. It is almost needless to say that I found all the Newtonian observations of these rings completely verified; but as his experiments seemed to be too much confined for drawing general conclusions, I endeavoured to extend them : and by way of rendering the methods I point out very clear, I have given one easy particular instance of each, with the addition of a generalization of it, as follows :

First Method. On a table placed before a window I laid down a slip of glass the sides of which were perfectly plain, parallel, and highly polished. Upon this I laid a double convex lens of 26 inches focal length, and found that this arrangement gave me a set of beautiful concentric rings.

I viewed them with a double convex eye lens of $2 \frac{1}{2}$ inches focus mounted upon an adjustable stand, by which simple apparatus I could examine them with great ease ; and as it was not material to my present purpose by what obliquity of incidence of light $I$ saw the rings, I received the rays

\footnotetext{
"Newton's Optics, thed, p. 288; end of Obs. 12.
} 
from the window most conveniently when they fell upon the lens in an angle of about 30 degrees from the perpendicular, the eye being placed on the opposite side at an equal angle of elevation to receive the reflected rays.

Generalization. Instead of a plain slip of glass, the plain side of a plaino-concave, or plano-convex lens of any focal length whatsoever may be used: and when the convex side of any lens is laid upon it, whatever may be the figure of the other surface, whether plain, concave, or convex, and whatever may be its focal length, a set of concentric rings will always be obtained. I have seen rings with lenses of all varieties of focus, from 170 feet down to one quarter of an inch. Even a common watch-glass laid upon the same plain surface will give them.

To ensure success, it is necessary that the glasses should be perfectly well cleaned from any adhering dust or soil, especially about the point of contact; and in laying them upon each other a little pressure should be used, accompanied at first with a little side motion, after which they must be left at rest.

If the surface of the incumbent lens, especially when it is of a very short focal length, is free from all imperfection and highly polished, the adjustment of the focus of the above-mentioned eye-glass, which $I$ always use for viewing the rings, is rather troublesome, in which case a small spot of ink made upon the lens will serve as an object for a sufficient adjustment to find the rings.

Second Method. Instead of the slip of glass, I laid down a well polished plain metalline mirror; and placing upon it the same 26 -inch double convex lens, I saw again a complete set of concentric rings.

It is singular that, in this case, the rings reflected from a bright metalline surface will appear fainter than when the same lens is laid on a surface of glass reflecting but little light; this may however be accounted for by the brilliancy of the metalline ground on which these faint rings are seen, the contrast of which will offuscate their feeble appearance.

Generalization. On the same metalline surface every variety of lenses may be laid, whatever be the figure of their 
upper surface, whether plain, concave, or convex, and whatever be their focal lengths, provided the lowest surface remains convex, and concentric rings will always be obtained; but for the reason mentioned in the preceding paragraph, very small lenses should not be used till the experimentalist has been familiarized with the method of seeing these rings, after which lenses of two inches focus, and gradually less, may be tried.

Third Method. Hitherto we have only used a plain surface upon which many sorts of glasses have been placed; in order therefore to obtain a still greater variety, I laid down a plano-convex lens of 15 inches focal length, and upon the convex surface of 'it I placed the 26 -inch double convex lens, which produced a complete set of rings.

Fourth Method. The same lens placed upon a convex metalline mirror of about 15 inches focal length gave also a complete set of rings.

Generalization. These two cases admit of a much greater variety than the first and second methods; for here the incumbent glass may have not only one, but both its surfaces of any fignre whatsoever ; whether plain, concave, or convex; provided the radius of concavity, when concave lenses are laid upon the convex surface of glass or metal, is greater than that of the convexity on which they are laid.

The figure of the lowest surface of the subjacent substance, when it is glass, may also be plain, concave, or convex ; and the curvature of its upper surface, as well as of the mirror, may be such as to give them any focal length, provided the radius of their convexities is less than that of the concavity of an incumbent lens; in all which cases complete sets of concentric rings will be obtained.

Fifth Method. Into the concavity of a double concave glass of 8 inches focal length I placed a 7 -inch double convex lens, and saw a very beautiful set of rings.

Sixth Method. Upon a 7-feet concave metalline mirror I placed the double convex 26 -inch lens, and had a very fine set of rings.

Generalization. With these two last methods, whatever may be the radius of the concavity of the subjacent surface, provided 
provided it be greater than that of the convexity of the incumbent glass ; and whatever may be the figure of the upper surface of the lenses that are placed upon the former, there will be produced concentric rings. The figure of the lowest surface of the subjacent glass may also be varied at pleasure, and still concentric rings will be obtained.

\section{Of seeing Rings by Transmission.}

The great variety of the different combinations of these differently figured glasses and mirrors will still admit of further addition, by using a different way of viewing the rings. Hitherto, the arrangement of the apparatus bas been such as to make them visible only by reflection, which is evident, because all the experiments that have been pointed out may be made by the light of a candle placed so that the angle of incidence and of reflection towards the eye of the observer may be equal. But Sir I. Newton has given us also an observation where he saw these rings by transmission, in consequence of which $I$ have again multiplied and varied the method of producing them that way, as follows :

First Method. On a slip of plain glass highly polished on both sides place the same double convex lens of 26 inches, which had already been used when the rings were seen by reflection. Take them both up together and hold them against the light of a,window, in which position the concentric rings will be seen with great ease by transmitted light. But as the use of an eye-glass will not be convenient in this situation, it will be uecessary to put on a pair of spectacles with glasses of 5,6 , or 7 inches focus, to magnify the rings in order to see them more readily.

Second Method. It would be easy to construct an apparatus for viewing the rings by transmission fitted with a proper eye-glass; but other methods of effecting the same purpose are preferable. Thus, if the two glasses that are to give the rings be laid upon a bollow stand, a candle placed at a proper angle and distance under them will show the rings conveniently by transmitted light, while the observer and the apparatus remain in the same situation as if they were to be seen by reflection. 
Third Method. A still more eligible way is to use daylight received upon a plain metalline nuirror reflecting it upwards to the glasses placed over it, as practised in the construction of the common double microscope : but I forbear entering into a further detail of this last and most useful way of seeing rings by transmission, as I shall soon have occasion to say more on the same subject.

Generalization. Every combination of glasses that has been explained in the first, third, and fifth methods of seeing rings by reflection will also give them by transmission, when exposed ito the light in any of the three ways that have now been pointed out. When these are added to the former, it will be allowed that we have an extensive variety of arrangements for every desirable purpose of making experiments upon rings, as far as single sets of them are concerned.

\section{Of Shadows.}

When two or more sets of rings are to be seen, it will require some artificial means, not only to examine them critically, but even to perceive them ; and here the shadow of some slender opaque body will be of eminent service. To east shadows of a proper size and upon places where they are wanted, a pointed penknife may be used as follows :

When a plain slip of glass or convex lens is laid down, and the point of a penknife is brought over either of them, it will cast two shadows, one of which may be seen on the first surface of the glass or lens, and the other on the lowest.

When two slips of glass are laid upon each other, or a convex lens upon one slip, so that both are in contact, the penknife will give three shadows; but if the convex lens should be of a very short focus, or the slips of glass a little separated, four of them may be perceived; for in that case there will be one formed on the lowest surface of the incumbent glass or lens; but in my distinction of shadows this will not be noticed. Of the three shadows thus formed the second will be darker than the first, but the third will be faint. When a piece of looking-glass is substituted for the lowest slip the third shadow will be the strongest.

Three slips of glass in contact, or two slips with a lens upon them, or also a looking glass, a slip and a lens put together, 
together, will give four shadows, one from each upper surface and one from the bottom of the lowest of them.

In all these cases a metalline mirror may be laid under the same arrangement without adding to the number of shadows, its effect being only to render them more intense and distinct.

The shadows may be distinguished by the following method: When the point of the penknife is made to touch the surface of the uppermost glass or lens, it will touch the point of its own shadow, which may thus at any time be easily ascertained : and this in all cases I call the first shadow; that which is next to it, the second; after which follows the third, and so on.

In receding from the point, the shadows will mix together, and thus become more intense; but which, or how mary of them are united together, may always be known by the points of the shadows.

When a shadow is to be thrown upon any required place, hold the penknife nearly half an inch above the glasses, and advance its edge foremost gradually towards the incident light. The front should be held a little downwards to keep the light from the underside of the penknife, and the shadows to be used should be obtained from a narrow part of it.

With this preparatory information it will be easy to point out the use that is to be made of the shadows when they are wanted.

\section{Of two Sets of Rings.}

I shall now procetd to describe a somewhat more complicated way of observation, by which two complete sets of concentric rings may be seen at once. The new or additional set will furnish us with an opportunity of examining rings in situations where they have never been seen before, which will be of eminent service for investigating the cause of their origin, and with the assistance of the shadows to be formed, as has been explained, we shall not find it difficult to see them in these situations.

First Method. Upon a well polished piece of good looking-glass lay down a double convex lens of about 20 inches focus. When the eye-glass has been adjusted as usual for 
seeing one set of rings, make the shadow of the penknife, in the order which has been described, pass over the lens; then, as it sometimes happens in this arrangement that no rings are easily to be seen, the shadow will, in its passage over the surface, show where they are situated. When 2 set of them is perceived, which is generally the primary one, bring the third shadow of the penknife over it, in which situation it will be seen to the greatest advantage.

Then, if at the same time a secondary set of rings has not yet been discovered, it will certainly be perceived when the second shadow of the penknife is brought upon the primary set. As soon as it has been found out, the compound shadow, consisting of all the three shadows united, may then be thrown upon this secondary set, in order to view it at leisure and in perfection. But this compound shadow should be taken no further from the point than is necessary to cover it; nor should the third shadow touch the primary set. The two sets are so near together, that many of the rings of one set intersect some of the other.

When a sight of the secondary set has been once obtained, it will be very easy to view it alternately with the primary one by a slight motion of the penknife, so as to make the third shadow of it go from one set to the other.

Besides the use of the shadows, there is another way to make rings visible when they cannot be easily perceived, which is to take hold of the lens with both hands, to press it alternately a little more with one than with the other; a tilting motion, given to the lens in this manner, will move the two sets of rings from side to side; and as it is well known that a faint object in motion may be sooner perceived than when it is at rest, both sets of rings will by these means be generally detected together.

It will also contribute much to facilitate the method of seeing two sets of rings, if we receive the light in a more oblique angle of incidence, such as 40,30 , or even 60 degrees. This will increase the distance between the centres of the primary and secondary sets, and at the same time occasion a more copious reflection of light.

Instead of a common looking-glass a convex glass mirror may 
may be used, on which may be placed either a plain, a concave, or a convex surface of any lens or glass, and two sets of rings will be obtained.

In the same manner, by laying upon a concave glass mirror a convex lens, we shall also have two sets of rings.

The generalizations that have been mentioned when one set of rings was proposed to be obtained, may bo easily applied with proper regulations, according to the circumstances of the case, not only to the method by glass mirrors already mentioned, but likewise to all those that follow hereafter, and need not be particularized for the future. In the choice of the surfaces to be joined, we have only to select such as will form a central contact, the focal length of the lenses and the figure of the upper surface being variable at pleasure.

Second Method. On a plain metalline mirror I laid a parallel slip of glass, and placed upon it the convex surface of a 17 -inch plano-convex lens, by which means two sets of rings were produced.

Upon the same mirror the plain side of the plano-convex glass may be laid instead of the plain slip, and any plain, convex, or concave surface being placed upon the convexity of the subjacent lens, will give two sets of rings.

The plain side of a plano-concave glass may also be placed upon the same mirror, and into the concavity may be laid any lens that will make a central contact with it, by which arrangement two sets of rings will be obtained.

Third Method. Upon a small well polished slip of glass place another slip of the same size, and upon them lay a 39-inch double convex lens. This will produce two sets of rings; one of them reflected from the upper surface of the first slip of glass, and the other from that of the second.

Instead of the uppermost plain slip of glass we may place upon the lowest slip the plain side of a plano-oonvex or plano-concave lens, and the same variety which has been explained in the third method, by using any incumbent lens that will make a central contact, either with the convexity or concavity of the subjacent glass, will always produce two sets of riings.

Vol. 30. No. 112. Fel. 1808.

Fourth 
Fourth Method. A more refined but rather more difficule way of seeing two sets of rings, is to lay a plain slip of glass on a piece of black paper, and when a convex lens is placed upon the slip, there may be perceived, but not without particular attention, not only the first set, which has already been pointed out as reflected from the first surface of the slip, but also a faint secondary set from the lowest surface of the same slip of glass.

It will be less difficult to see two sets of rings by a reflection from both surfaces of the same glass, if we use, for instance, a double concave of 8 inches focus with a double convex of $7 \frac{1}{2}$ inches placed upon it. For, as it is well known that glass will reflect more light from the furthest surface when air rather than a denser medium is in contact with it, the hollow space of the 8 -inch concave will give a pretty strong reflection of the secondary set.

Fiflh Method. The use that is intended to be made of two sets of rings requires that one of them should be dependent upon the other: this is a circumstance that will be explained hereafter; but the following instance, where two independent sets of rings are given, will partly anticipate the subject. When a double convex lens of 50 inches is laid down with a slip of glass placed upon it, and another double convex one of 26 inches is then placed upon the slip, we get two sets of rings of djfferent sizes; the large rings are from the 50 -inch glass, the small rings from the 26 -inch one. They are to be seen with great ease, because they are each of them primary. By tilting the incumbent lens or the slip of glass these two sets of rings may be made to cross each other in any direction; the small set may be laid upon the large one, or either of them may be separately removed towards any part of the glass. This will be sufficient to show that they have no connection with each other. The phrnomena of the motions, and of the various colours and sizes assumed by these rings, when different pressures and tiltings of the glasses are used, will afford some entertainment. With the assistance of the shadow of the penknife the secondary set belonging to the rings from the 26-inch lens will be added to the other two 
sets; but in tilting the glasses this set will never leave its primary one, while that from the 50 -inch lens may be made to go any where across the other two.

\section{Of three Sets of Rings.}

To see three sets of concentric rings at once is attended with some difficulty; but by the assistance of the methods of tilting the glasses and making use of the multiplied shadows of a penknife, we may see them very well, when ther is a sufficient illumination of bright daylight.

First Method. A 26 -inch double convex lens placed upon three slips of plain glass, will give three sets of rings, The slips of glass should be nearly 2-tenths of an inch thick, otherwise the different sets will not be sufficiently separated. When all the glasses are in full contact, the first and second sets may be seen with a little pressure and a small motion, and, if circumstances are favourable, the third, which is the faintest, will also appear. If it cannot be seen, some of the compound shadows of the penknife must be thrown upon it ; for in this case there will be five shadows visible, several of which will fall together and give different. intensity to their mixture.

Second Method. When a single slip of glass, with a 3.1inch lens upon it, is placed upon a piece of good lookingglass, three sets of rings may be seen: the first and third sets are pretty bright, and will be perceived by only pressing the lens a little upon the slip of glass; after which it will be easy to find the second set with the assistance of the proper shadow. In this case four shadows will be seen; and when the third shadow is upon the first set, the fourth will be over the second set and render it visible.

Third Method. When two slips of glass are laid upon a plain metalline mirror, then a 26 -inch lens placed upon the slips will produce three sets of rings; but it is not very easy to perceive them. By a tilting motion the third set will generally appear like a small white circle, which at a proper distance will follow the movement of the first set. As soon as the first and third sets are in view, the third shadow of the penknife may be brought over the first set, by which means 
the fourth shadow will come upon the second set, and in this position of the apparatus it will become visible.

Fourth Method. On a plain metalline mirror lay one slip. of glass, but with a small piece of wood at one end under it, so that it may be kept about one-tenth of an inch from the mirror, and form an inclined plane. A 96 -inch lens laid upon the slip of glass will give three scts of rings. Two of them will easily be seen; and when the shadow of the penknife is held between them, the third set will also be perceived. There is but one shadow visible in this arrangement, which is the third; the first and second shadows being lost in the bright reflection from the mirror.

Fifth Method. I placed a $6 \frac{\mathrm{a}}{4}-$ inch double convex upon an 8-inch double concave, and laid both together upon a plain slip of glass. This arrangement gave three sets of rings. They may be seen without the assistance of shadows, by using only pressure and tilting. The first had a black and the other two had white centres.

\section{Of four Sets of Rings.}

The difficulty of seeing many sets of rings increases with their number, yet by a proper attention to the directions that are given, four sets of concentric rings may be seen.

First Method. Let a slip of glass, with a 96 -inch lens laid upon it, be placed upon a piece of looking-glass. Under one end of the slip, a small piece of wood one-tentb of an inch thick must be put to keep it from touching the lookingglass. This arrangement will give us four sets of rings. The first, third, and fourth may easily be seen, but the second ser will require some management. Of the three shadows which this apparatus gives, the second and third must be brought between the first and fourth sets of rings, in which situation the second set of rings will become visible.

Second Method. When three slips of glass are laid upon a metalline mirror, and a plano-convex lens of about 17 inches focus is placed with its convex side upon them, four sets of rings may be seen; but this experiment requires a very bright day, and very clean, highly polished slips of plain glass. Nor can it be successful unless all the foregoing 
going methods of seeing multiplied sets of rings are become familiar and easy.

I have seen occasionally, not only four and five, but even six sets of concentric rings, from a very simple arrangement of glasses: they arise from reiterated internal reflections; but it will not be necessary to carry this account of seeing multiplied sets of rings to a greater length.

\section{Of the Size of the Rings.}

The diameter of the concentric rings depends upon the radius of the curvature of the surfaces between which they are formed. Curvatures of a short radius, cateris parilus, give smaller rings than those of a longer; but sir Isaac Newton having already treated on this part of the subject at large, it will not be necessary to enter further into it.

I should however remark, that when two curves are concerned, it is the application of them to each other that will determine the size of the rings, so that large ones may be produced from curvatures of a very short radius. A double convex lens of $2 \frac{1}{4}$ inches focus, for instance, when it is laid upon a double concave which is but little more in focal length, gives rings that are larger than those from a lens of 26 inches laid upon a plain slip of glass.

\section{Of Contact.}

The size of the rings is considerably affected by pressure. They grow larger when the two surfaces that form them are pressed closer together, and diminish when the pressure is gradually removed. The smallest ring of a set may be increased by this means to double and treble its former diameter; but as the common or natural pressure of glasses laid upon any flat or curved surface is occasioned by their weight, the variations of pressure will not be very considerable when they are left to assume their own distance or contact. To produce that situation, however, which is generally called contact, it will always be necessary to give a little motion backwards and forwards to the incumbent lens or glass, accompanied with some moderate pressure, after which it may be left to settle properly by its own weight. 


\section{Of measuring Rings.}

It may be supposed from what has been said concerning the kind of contact which is required for glasses to produce rings, that an attempt to take absolute measures must be liable to great inaccuracy. This was fully proved to me when I wanted to ascertain, in the year 1792 , whether a lens laid upon a metalline surface would give rings of an equal diameter with those it gave when placed on glass. The measurcs differed so much that I was at first deceived; but on proper consideration it appeared that the Huygenian object glass, of 122 feet foeus, which I used for the experiment, could not so easily be brought to the same contact on metal as on glass; nor can we ever be well assured that an equal distance between the two surfaces in both cases has been actually obtained. The colour of the central point, as will be shown hereafter, may serve as a direction; bul even that cannot be easily made equal in both cases. By taking a sufficient number of measures of any given ring of a set, when a glass of a sufficient focal length is used, we may however determine its diameter to about the 25th or 30th part of its dimension.

Relative measures for ascertaining the proportion of the different rings in the same set to each other, may be more accurately taken; for in that case the contact with them all will remain the same, if we do not disturb the glasses during the time of measuring.

\section{Of the Number of Rings.}

When there is a sufficient illumination, many concentric rings in every set will be perceived; in the primary set we see generally 8, 9, or 10, very conveniently. By bolding the eye in the most favourable situation $I$ have often counted near 20 , and the number of them is generally lost when they grow too narrow and minute to be perceived, so that we can never be said fairly to have counted them to their full extent. In the second set I have seen as many as in the first, and they are full as bright. The third set, when it is seen by a metalline mirror under two slips, will be brighter than the second, 
second, and almost as bright as the first: I have easily counted 7,8 , and 9 rings.

\section{Of the Effect of Pressure on the Colour of the Rings.}

When a double convex object glass of 14 or 15 feet focus is laid on a plain slip of glass, the first colours that make their faintest appearance will be red surrounded by green; the smallest pressure will turn the centre into green surrounded by red: an additional pressure will give a red centre again, and so on till there have been so many successive alterations as to give us six or seven times a red centre, after which the greatest pressure will only produce a very large black one surrounded by white.

When the rings are seen by transmission, the colours are in the same manner subject to a gradual alternate change occasioned by pressure; but when that is carried to its full extent, the centre of the rings will be a large white spot surrounded by black.

The succession and addition of the other prismatic colours after the first or second change, in both cases is extremely beautiful; but as the experiment may be so easily made, a description, which certainly would fall short of an actual view of these phænomena, will not be necessary.

When the rings are preduced by curves of a very short radius, and the incumbent lens is in full contact with the slip of glass, they will be alternately black and white; but by lessening the contact, I have seen, even with a double convex lens of no more than two-tenths of an inch focus, the centre of the rings white, red, green, yellow, and black, at pleasure. In this case I used an eye-glass of one inch focus; but as it requires much practice to manage such small glasses, the experiment may be more conveniently made by placing a double convex lens of two-inch focus on a plain slip of glass, and viewing the rings by an eye-glass of $2 \frac{1}{2}$ inches; then having first brought the lens into full contact, the rings will be only black and white, but by gently lifting up or tilting the lens, the centre of the rings will assume various colours at pleasure. 
XII. Of diluting and concentrating the Colours.

Lifting up or tilting a lens being subject to great uncerfainty, a surer way of acting upon the colours of the rings is by dilution and concentration. After having seen that vẹry small lenses give only black and white when in full contact, we may gradually take others of a longer focus. With a double convex lens of four inches the outward rings will begin to assume a faint red colour. With 5,6 , and 7 , this appearance $u$ ill increase; and proceeding with lenses of a larger focus, when we come to about 16,18 , or 20 inches, green rings will gradually make their appearance.

This and other colours come on much sooner if the centre of the lens is not kept in a black contact, which in these experiments must be attended to.

A lens of 26 inches not only shows black, white, red, and green rings, but the central black begins already to be diIuted so as to incline to violet, indigo, or blue. With one of 34 , the white about the dark centre begins to be diluted, and shows a kind of gray inclining to yellow. With 42 and 48 , yellow rings begin to become visible. With 55 and 59 , blue rings show themselves very plainly. With a focal length of 9 and 11 feet, orange may be distinguished from the yellow, and indigo from the blue. With 14 feet, some violet becomes visible. When the 122 feet Huygenian glass is laid on a plain slip, and well settled upon it, the central colour is then sufficiently diluted to show that the dark spot, which in small lenses, when concentrated, had the appearance of black, is now drawn out into violet, indigo, and blue, with little admixture of green ; and that the white ring, which used to be about the central spot, is turned partly green with a surrounding yellow, orange, and red-coloured space or ring; by which means we seen to have a fair analysis of our former compound black and white centre.

One of my slips of glass, which is probably a little concave, gave the rings still larger when the 122 feet glass was firmly pressed against it. I used a little side motion at the same time, and brought the glasses into such contact that they adhered sufficiently to be lifted up together. With this adhesion 1 perceived a colour surrounding a dark 
centre which $I$ have never seen in any prismatic spectrum. It is a kind of light brown, resembling the colour of a certain sort of Spanish snuff. The 170 feet object glass showed the same colour also very clearly.

\section{Of the Order of the Colours.}

The arrangement of the colours in each compound ring or alternation, seen by reflection, is, that the most refrangible rays are nearest the centre; and the same order takes place when seen by transmission. We have already shown that when a full dilution of the colours was obtained their arrangemont was violet, indigo, blue, green, yellow, orange and red; and the same order will hold good when the colours are gradually concentrated again; for though some of them should vanish before others, those that remain will always be found to agree with the same arrangement.

If the rings should chance to be red and green alternately, $a$ doubt might arise which of them is nearest the centre; but by the method of dilution, a little pressure, or some small increase of the focal length of the incumbent lens, there will be introduced an orange tint between them, which will immediately ascertain the order of the colours.

In the second set of rings the same order is still preserved as in the first ; and the same arrangement takes place in the third set as well as in the fourth. In all of them the most refrangible rays produce the smallest rings.

XIV. Of the alternate Colour and Size of the Rings velonging to the primary and dependent Sets.

When two sets of rings are seen at once, and the colour of the centre of the primary set is black, that of the secondary will be white; if the former is white, the latter will be black. The same alternation will take place if the colour of the centre of the primary set should be red or orange; for then the centre of the secondary one will be green ; or if the former happens to be green, the latter will be red or orange. At the same time there will be a similar alternation in the size of rings ; for the white rings in one set will be of the diameter of the black in the other; or the orange rings of the former will be of equal magnitude with the green of the latter.

When three sets of rings are to be seen, the second and 
90 Surgical Cases in the City and Finslury Dispensaries.

third sets will be alike in colour and size, but alternate in both particulars with the primary set.

The same thing will happen when four sets are visible; for all the sets that are formcd from the primary one will resemble each other, but will be alternate in the colour and dimensions of their rings with those of the primary set.

['To be continued.]

XIX. Report of Surgical Cases in the City and Finslury Dispensaries for September 1807. By Joн N Ta unton, Esq. $I_{N}$ the month of September there were admitted on the books of the City and Finsbury Dispensaries 237 surgical patients.

$$
\begin{array}{llllr}
\text { Cured or relieved } & - & - & 205 \\
\text { Died } & - & - & - & 4 \\
\text { Under cure } & - & - & - & 28 \\
\hline
\end{array}
$$

Since which time there have been admitted 963 .

One of the fatal cases was that of Mrs. M. S. æt. 67, who, soon after the birth of her ninth and last child, was seized with femoral hernia : the tumour was small, but painful, attended with sickness and inclination to vomit; these symptoms did not last long, as the hernia receded without assistance: she had another attack in about six weeks, which was also of short duration; the hernia soon became irreducible, and she had been exposed to similar attacks for the last sixteen years of her life, for which time it had never been completely returned: these were occasionally longer and more severe; the symptoms frequently continued for two or three days, but were removed without any operation or medicine, except some Daffy's elixir.

On the $22 \mathrm{~d}$ of August she was seized with the usual symptoms, viz. pain over the whole belly, particularly in the region of the stomach, with a sensation of heat, somiting, cold sweats, intermitting pulse, and suppression of stools for four days: the tumour was now much larger than before, being nearly the size of a child's head at birth : at this period she had a motion of a small quantity of feculent 